\title{
THE EFFICIENGY OF THE GAMMA APPROXIMATION OF BAYESIAN ESTIMATION OF THE ERROR VARIANGE OF THE SECOND ORDER MOVING AVERAGE PROCESS
}

\section{MAJDI AMEEN KUTBI}

Department of Mathematical Sciences

Faculty of Applied Sciences

Umm Al-Qura University

Makkah

Saudi Arabia

makutbi@uqu.edu.sa 


\section{ABSTRACT}

The main objective of this article is to examine the numerical efficiency of the gamma approximation technique, developed by Broemeling and Shaarawy (1988), to estimate the error variance of the noise term of the second order moving average process. In order to achieve our main goal, six simulation studies are conducted with different variances and coefficients ; then proposed criteria are calculated. The inspection of the numerical results shows that the proposed approximation can efficiently estimate the noise variance with very high precision for moderate and large time series lengths. In addition, the numerical results show that better results can be obtained if the error variance is small.

Keywords : Moving average processes ; Error variance ; Likelihood function ; Jeffreys' prior ; Posterior density function; Simulation .

\section{INTRODUGTION}

The literature on time series is vast and may be found in many areas other than statistics such as economics, business, physics, engineering and environmental studies. Most of the literature is non-Bayesian and the reader is referred to Box and Jenkins (1970), Chatfield (1980), Priestely (1981), Harvey (1993), Box et.al (2008), Wei (2005) and Liu (2009) for the non-Bayesian theory and methodology.

Most of Bayesian contributions of time series focus on pure autoregressive processes and pay little attention to moving average processes, denoted by MA(q), or mixed ARMA processes. The difficulty with $\mathrm{MA}(\mathrm{q})$ processes is that the likelihood function is analytically intractable and statistical inferences should be done numerically. Zellner (1971) introduced the reader to the Bayesian analysis of pure autoregressive 
processes. He used Jeffreys' vague prior to develop the posterior distributions of the first and second orders autoregressive models. Newbold (1973) did a Bayesian analysis of transfer function processes of which the ARMA processes are special case. Macleod (1977) proposed replacing the determinant of the covariance matrix of ARMA processes by its asymptotic limit in order to approximate the conditional likelihood function. However, his approach does not avoid the problem of computing the inverse of the covariance matrix. Phadke and Kedem (1978) presented three different techniques to obtain the exact likelihood function for moving average processes. However, none of these techniques avoid the problem of computing the inverse of the covariance matrix. Zellner and Reynold (1978) showed that the statistical inferences about the coefficients can be approximately done using a non-central multivariate $t$ distribution. Monahan (1983) made a very important contribution to time series analysis. Using a numerical integration technique, he implemented the identification, estimation and forecasting phases of ARMA processes. Lahif (1980) investigated the first autoregressive process in the same way Monahan did with mixed models. Shaarawy and Broemeling (1984) presented an approximate methodology to estimate the parameters of pure moving average processes. They showed that the posterior distribution of the precision parameter can be approximated by a gamma distribution. Broemeling and Shaarawy $(1986,1988)$ did a comprehensive approximate technique to implement the identification, estimation, diagnostic checking and forecasting phases of ARMA processes. The numerical efficiency of their estimation technique has been investigated by Shaarawy and ElShawadfy (1994) for the coefficients only. Kutbi(2010) examined the efficiency of their approximation for the precision parameter of the first order moving average process. However, the efficiency of their technique to estimate the error variance has not been investigated yet. 
The essential objective of this paper is to study and investigate the behavior of the gamma approximation , proposed by Shaarawy and Broemeling(1984), in estimating the variance parameter of the error term of the second order moving average processes. The performance of the gamma approximation is investigated through a large simulation study. Section 2 of this paper presents the second order moving average processes and shows how to use the gamma approximation to estimate the error variance. Section 3 is focused to conduct six simulation studies to investigate the numerical efficiency of the gamma approximation which will be denoted by the BS technique. Finally, Section 4 presents the summary and conclusions of the research paper.

\section{MOVING AVERAGE PROGESS OF THE SECOND ORDER}

The Bayesian analysis of time series is based on special parametric models such as regression models with autocorrelated errors , distributed lag model, autoregressive models , and moving average models. A very important model is the second order moving average process, denoted by $\operatorname{MA}(2)$, which is defined by :

$$
\mathrm{y}(\mathrm{t})=\Theta(\mathrm{B}) \varepsilon(\mathrm{t})
$$

where $y(t)$ is the observation at time $t=\ldots \ldots .,-2,-1,0,1,2, \ldots$. , $\varepsilon(\mathrm{t})$ is a sequence of independent normally distributed random variables with mean zero and unknown precision $\tau=1 / \sigma^{2}>0$. The polynomial operator $\Theta(B)$ is given by :

$$
\Theta(B)=1-\theta_{1} B-\theta_{2} B^{2}
$$

where $\theta_{1}$ and $\theta_{2}$ are real unknown constants, and the backshift operator $\mathrm{B}$ is such that : 


$$
\mathrm{B}^{\mathrm{r}} \varepsilon(\mathrm{t})=\varepsilon(\mathrm{t}-\mathrm{r}) \quad, \quad \mathrm{r}=1,2
$$

The MA(2) process is always stationary and is invertible if the roots of the equation $\Theta(B)=0$ lie outside the unit circle. It can be shown that the $\operatorname{MA}(2)$ process is invertible if $\theta_{1}+\theta_{2}<1$, $\theta_{2}-\theta_{1}<1$ and $\left|\theta_{2}\right|<1$, see Box and Jenkins(1970). The main problem with analyzing the $\mathrm{MA}(2)$ process is that the sum of squared errors is nonlinear function of the coefficients $\theta_{1}$ and $\theta_{2}$.

Suppose $\mathrm{n}$ observations are available, say

$$
\mathrm{S}_{\mathrm{n}}=\mathrm{y}(1), \mathrm{y}(2), \ldots . ., \mathrm{y}(\mathrm{n})
$$

then the conditional likelihood function of the MA(2) process is

$$
\mathrm{L}\left(\underset{\sim}{\theta}, \tau \mid \underset{\sim}{\mathrm{S}_{\mathrm{n}}}\right) \propto \tau^{\mathrm{n} / 2} \operatorname{Exp}\left\{-\frac{\tau}{2} \sum_{\mathrm{t}=1}^{\mathrm{n}}\left[\mathrm{y}(\mathrm{t})-\theta_{1} \varepsilon(\mathrm{t}-1)-\theta_{2} \varepsilon(\mathrm{t}-2)\right]^{2}\right\}
$$

where $\underset{\sim}{\theta}=\left(\theta_{1}, \theta_{2}\right) \in \mathrm{R}^{2}, \tau>0 \quad$ and $\quad \varepsilon(\mathrm{t}-2)=0, \mathrm{t}=1,2$. Thus the likelihood is conditional on the first 2 errors being zero. The maximum likelihood estimators of the parameters $\theta_{1}$ and $\theta_{2}$ is equivalent to minimizing the conditional sum of squared errors. However, the error of the MA(2) model can be written as

$$
\varepsilon(\mathrm{t})=\mathrm{y}(\mathrm{t})+\theta_{1} \varepsilon(\mathrm{t}-1)+\theta_{2} \varepsilon(\mathrm{t}-2) \quad, \quad \mathrm{t}=1,2, \ldots, \mathrm{n}
$$

which can be computed recursively if one knew $\underset{\sim}{\theta}$. Since the sum of squared errors, 


$$
\mathrm{SS}(\underset{\sim}{\theta})=\sum_{\mathrm{t}=1}^{\mathrm{n}}\left[\mathrm{y}(\mathrm{t})+\theta_{1} \varepsilon(\mathrm{t}-1)+\theta_{2} \varepsilon(\mathrm{t}-2)\right]^{2}
$$

is a nonlinear function of $\underset{\sim}{\theta}$, one must use a grid search ( usually over the invertibility domain ) or employ a nonlinear regression algorithm in order to estimate $\underset{\sim}{\theta}$. The maximum likelihood estimate $\underset{\sim}{\theta}$ my be used to calculate the estimated residuals, via equation (2.3), with $\varepsilon(\mathrm{t}-2)=0, \mathrm{t}=1,2$, producing

$$
\hat{\varepsilon}(t)=y(t)+\sum_{j=1}^{2} \hat{\theta}_{j} \hat{\varepsilon}(t-j)
$$

If these estimated errors are substituted in to (2.2), one has an approximate conditional likelihood function :

$$
\mathrm{L}^{*}\left(\underset{\sim}{\theta}, \tau \mid \mathrm{S}_{\mathrm{n}}\right) \propto \tau^{\mathrm{n} / 2} \operatorname{Exp}\left\{-\frac{\tau}{2} \sum_{\mathrm{t}=1}^{\mathrm{n}}\left[\mathrm{y}(\mathrm{t})+\theta_{1} \hat{\varepsilon}(\mathrm{t}-1)+\theta_{2} \hat{\varepsilon}(\mathrm{t}-2)\right]^{2}\right\}
$$

where $\theta \in R^{2}, \tau>0$, and $S_{n}$ is the vector of $n$ observations.

The posterior analysis is based on combining the approximate conditional likelihood function (2.4) with a normal-gamma prior density where $\underset{\sim}{\theta} \mid \tau \sim \underset{\sim}{\mathrm{N}}(\underset{\mu}{\mu}, \mathrm{P} \tau)$ is the conditional prior density of $\underset{\sim}{\theta}$ given $\tau$, where $\mu$ is $2 \times 1$ vector and $\mathrm{P}$ a $2 \times 2$ positive definite matrix. The marginal prior density of $\tau$ is gamma with parameters $a$ and $b$. Thus the joint posterior distribution of $\underset{\sim}{\theta}$ and $\tau$ will also be normal gamma.

Shaarawy and Broemeling(1984) have shown that the approximate marginal posterior distribution of $\tau$ is gamma with parameters 


$$
\alpha=\frac{(\mathrm{n}+2 \mathrm{a})}{2}
$$

and

$$
\beta=\left(\mathrm{C}-\underset{\sim}{\mathrm{B}^{\prime}} \mathrm{A}^{-1} \underset{\sim}{\mathrm{B}}\right) / 2
$$

where $\mathrm{A}$ is the $2 \times 2$ matrix

$$
A=P+\left(a_{i j}\right),
$$

$\underset{\sim}{\mathrm{B}}$ is a $2 \times 1$ vector

$$
\underset{\sim}{\mathrm{B}}=\underset{\sim}{\mathrm{P}}-\left(\mathrm{b}_{\mathrm{j}}\right)
$$

and $\mathrm{C}$ is the scalar,

$$
\mathrm{C}=\underset{\sim}{\mu^{\prime}} \mathrm{P} \underset{\sim}{\mu}+2 \mathrm{~b}+\sum_{\mathrm{t}=1}^{\mathrm{n}} \mathrm{y}^{2}(\mathrm{t})
$$

Further more

$$
\left(a_{i j}\right)=\sum_{t=1}^{n} \hat{\varepsilon}(t-i) \hat{\varepsilon}(t-j) \quad, \quad i, j=1,2
$$

and

$$
\left(b_{j}\right)=\sum_{t=1}^{n} y(t) \hat{\varepsilon}(t-j) \quad, \quad j=1,2
$$

Thus, one may estimate the error variance $\sigma^{2}=\tau^{-1}$ by

$$
\mathrm{E}\left(\sigma^{2} \mid \mathrm{S}_{\mathrm{n}}\right)=\mathrm{E}\left(\tau^{-1} \mid \mathrm{S}_{\mathrm{n}}\right)=\frac{\beta}{\alpha-1}
$$


with posterior variances given by

$$
\mathrm{V}\left(\sigma^{2} \mid \mathrm{S}_{\mathrm{n}}\right)=\mathrm{V}\left(\tau^{-1} \mid \mathrm{S}_{\mathrm{n}}\right)=\frac{\beta^{2}}{(\alpha-1)^{2}(\alpha-2)}
$$

The above posterior analysis is derived under the assumption that a normal - gamma describes prior information. Thus, if one is quite confident about the prior distribution of the parameter, one would specify such prior by the appropriate choice of $\underset{\sim}{\mu}, \mathrm{P}$, $\mathrm{a}$, and $\mathrm{b}$, however , if one knows very little, a priori, about the parameters $\underset{\sim}{\theta}$ and $\tau$, one might employ Jeffrey's vague density :

$$
\xi(\underset{\sim}{\theta}, \tau) \propto \tau^{-1} \quad, \quad \underset{\sim}{\theta} \in \mathrm{R}^{2}, \tau>0
$$

which will produce a normal - gamma posterior density with parameters given above, by letting

$$
\underset{\sim}{\mu} \rightarrow 0(2 \times 1), \mathrm{P} \rightarrow 0(2 \times 2), \mathrm{a} \rightarrow-1, \text { and } \mathrm{b} \rightarrow 0 .
$$

\section{AN EFFECTIUENESS STUDY}

This main objective of this section is to study the performance of the gamma approximation in estimating the variance of the error term of the moving average processes of the second order. The approximation, which has been developed by Broemeling and Shaarawy(1988), is used with Jeffrey's prior density to conduct a simulation study to estimate the parameter $\sigma^{2}$ of MA(2) model with various parameter values. The parameters in some cases are chosen to be well inside the invertibilility domain while in some cases they are chosen to be near the boundaries. All computations are performed on a PC using the MATLAB computer package . 
Here, our main concern is to study the numerical efficiency of the gamma approximation, proposed by Broemeling and Shaarawy(1988) for the precision parameter, in estimating the error variance $\sigma^{2}$ using some proposed criteria. The proposed criteria are the average (AVR), the variance (VAR), the mean absolute deviation (MAD) and the mean squared error (MSE). Such efficiency will be examined with respect to the time series length $\mathrm{n}$ as well as the parameters of the selected model.

Simulation I , as an illustration, begins with generating 500 data sets, each of size 500 , from normal distribution with zero mean and variance $\sigma^{2}=0.5$. These data sets are then used to generate 500 realizations, each of size 200 , from MA(2) process with $\theta_{1}=0.5$ and $\theta_{2}=-0.6$. Note that the first 300 observations are ignored to remove the initialization effect. The second step of simulation I is to carry out all computations required to estimate the variance parameter $\sigma^{2}$ using each of the 500 realizations and to find the frequency distribution of the posterior mean of $\sigma^{2}$. Such computations are done for a specific time series length $n$ using the first $\mathrm{n}$ observations of each generated realization. This second step is repeated for each chosen sample size. The sample size $\mathrm{n}$ is taken to be $20,30,50,100,150$ and 200 . The frequency distribution of the error variance is reported in table 1. The average (AVR), the variance (VAR), the mean absolute deviation (MAD) and the mean squared error (MSE) are computed for the posterior mean and reported in table 2 . 
Table (1)

Frequency Distributions of The Posterior Expectation For Simulation I where :

$$
\sigma^{2}=0.5, \theta_{1}=0.5, \theta_{2}=-0.6
$$

\begin{tabular}{|c|c|c|c|c|c|c|}
\hline Intervals & $\mathbf{n}=\mathbf{2 0}$ & $\mathbf{3 0}$ & $\mathbf{5 0}$ & $\mathbf{1 0 0}$ & $\mathbf{1 5 0}$ & $\mathbf{2 0 0}$ \\
\hline $\mathbf{( - 0 . 6 , - 0 . 5}]$ & 1 & 1 & 1 & 1 & 1 & 1 \\
\hline $\mathbf{( - 0 . 5 , - 0 . 4 ]}$ & 0 & 0 & 0 & 0 & 0 & 0 \\
\hline $\mathbf{( - 0 . 4 , - 0 . 3 ]}$ & 0 & 0 & 0 & 0 & 0 & 0 \\
\hline $\mathbf{( - 0 . 3 , - 0 . 2 ]}$ & 0 & 0 & 0 & 0 & 0 & 0 \\
\hline $\mathbf{( - 0 . 2 , - 0 . 1 ]}$ & 0 & 0 & 0 & 0 & 0 & 0 \\
\hline $\mathbf{( - 0 . 1 , 0 . 0 ]}$ & 2 & 0 & 0 & 0 & 0 & 0 \\
\hline $\mathbf{( 0 . 0 , 0 . 1}]$ & 6 & 0 & 0 & 0 & 0 & 0 \\
\hline $\mathbf{( 0 . 1 , 0 . 2 ]}$ & 15 & 0 & 0 & 0 & 0 & 0 \\
\hline $\mathbf{( 0 . 2 , 0 . 3 ]}$ & 48 & 33 & 13 & 0 & 0 & 0 \\
\hline $\mathbf{( 0 . 3 , 0 . 4}]$ & 105 & 106 & 73 & 43 & 25 & 9 \\
\hline $\mathbf{( 0 . 4 , 0 . 5}]$ & 106 & 157 & 183 & 221 & 220 & 255 \\
\hline $\mathbf{( 0 . 5 , 0 . 6}]$ & 104 & 104 & 153 & 196 & 234 & 224 \\
\hline $\mathbf{( 0 . 6 , 0 . 7 ]}$ & 49 & 59 & 62 & 38 & 21 & 11 \\
\hline $\mathbf{( 0 . 7 , 0 . 8}]$ & 29 & 27 & 11 & 2 & 0 & 1 \\
\hline $\mathbf{( 0 . 8 , 0 . 9}]$ & 21 & 12 & 3 & 0 & 0 & 0 \\
\hline $\mathbf{( 0 . 9 , 1 . 0 ]}$ & 13 & 2 & 2 & 0 & 0 & 0 \\
\hline $\mathbf{( 1 . 0 , 1 . 1}]$ & 1 & 0 & 0 & 0 & 0 & 0 \\
\hline $\mathbf{( 1 . 1 , 1 . 2}]$ & 0 & 0 & 0 & 0 & 0 & 0 \\
\hline $\mathbf{( 1 . 2 , 1 . 3 ]}$ & 0 & 0 & 0 & 0 & 0 & 0 \\
\hline $\mathbf{( 1 . 3 , 1 . 4}]$ & 0 & 0 & 0 & 0 & 0 & 0 \\
\hline $\mathbf{( 1 . 4 , 1 . 5}]$ & 0 & 0 & 0 & 0 & 0 & 0 \\
\hline $\mathbf{( 1 . 5 , 1 . 6}]$ & 0 & 0 & 0 & 0 & 0 & 0 \\
\hline
\end{tabular}

Inspecting the numerical results of table 1 , one may observe that the values of the posterior mean of the error variance $\sigma^{2}$ tends to fall in the interval $(0.4,0.6)$ with a midpoint 0.5 which the true value of our parameter. This becomes more clear as $n$ gets bigger. This means that the posterior mean tends to converge to 
the true parameter value $\sigma^{2}=0.5$. It might be important to notice that the percentages of the values of the posterior expectations which fall in the interval $(0.4,0.6)$ are $42 \%, 52.2 \%, 67.2 \%, 83.4 \%$, $90.8 \%$, and $95.8 \%$ for $\mathrm{n}=20,30,50,100,150$ and 200 . Thus one may conclude that the performance of the BS approximation is very good with moderate and large sample size.

Table 2 gives the numerical behavior of the proposed criteria AVR, VAR, MAD and MSE for the BS technique. The table shows an increasing trend in the efficiency of the BS technique in estimating the variance of the second order moving average process. The AVR converges to the true parameter 0.5. The VAR, MAD and MSE decrease as the sample size increases. The VAR decrease from 0.036 to 0.003 as $\mathrm{n}$ increases from 20 to 200 . The MAD decreases from 0.15 to 0.04 when $n$ increases from 20 to 200. Finally, the MSE decreases from 0.04 to 0.003 as $n$ increases from 20 to 200 .

Table (2)

The Behavior of The Average, Variance, The Mean Absolute Deviation and Mean square Error of The Bayesian estimates of Simulation I Where $\sigma^{2}=0.5, \theta_{1}=0.5, \theta_{2}=-0.6$

\begin{tabular}{|c|c|c|c|c|}
\hline $\mathbf{n}$ & AVR & VAR & MAD & MSE \\
\hline $\mathbf{2 0}$ & 0.480136 & 0.035699 & 0.150930 & 0.037610 \\
\hline $\mathbf{3 0}$ & 0.488125 & 0.020590 & 0.110658 & 0.019253 \\
\hline $\mathbf{5 0}$ & 0.497729 & 0.011523 & 0.084643 & 0.011538 \\
\hline $\mathbf{1 0 0}$ & 0.498959 & 0.005354 & 0.059293 & 0.005374 \\
\hline $\mathbf{1 5 0}$ & 0.500302 & 0.003499 & 0.046717 & 0.003345 \\
\hline $\mathbf{2 0 0}$ & 0.499137 & 0.002581 & 0.040323 & 0.002527 \\
\hline
\end{tabular}

The numerical results of simulations II and III where $\sigma^{2}=1.0$ and 2.0 respectively, with $\theta_{1}=0.5$ and $\theta_{2}=-0.6$, are explained in a similar way. The results are given by tables 3-6. 
The results of the simulations IV , V and VI where $\sigma^{2}=0.5,1.0,2.0$, respectively, with $\theta_{1}=0.5$ and $\theta_{2}=0.3$, are reported in tables 7-12. It might be important to mention that the numerical results get better when the true variance gets small .

\section{Table (3)}

Frequency Distributions of The Posterior Expectation For Simulation II where :

$$
\sigma^{2}=1.0, \theta_{1}=0.5, \theta_{2}=-0.6
$$

\begin{tabular}{|c|c|c|c|c|c|c|}
\hline Intervals & $\mathbf{n}=\mathbf{2 0}$ & $\mathbf{3 0}$ & $\mathbf{5 0}$ & $\mathbf{1 0 0}$ & $\mathbf{1 5 0}$ & $\mathbf{2 0 0}$ \\
\hline $\mathbf{( - 0 . 3 , - 0 . 2}]$ & 1 & 0 & 0 & 0 & 0 & 0 \\
\hline $\mathbf{( - 0 . 2 , - 0 . 1 ]}$ & 0 & 0 & 0 & 0 & 0 & 0 \\
\hline $\mathbf{( - 0 . 1 , 0 . 0}]$ & 2 & 0 & 0 & 0 & 0 & 0 \\
\hline $\mathbf{( 0 . 0 , 0 . 1}]$ & 0 & 0 & 0 & 0 & 0 & 0 \\
\hline $\mathbf{( 0 . 1 , 0 . 2}]$ & 4 & 0 & 0 & 0 & 0 & 0 \\
\hline $\mathbf{( 0 . 2 , 0 . 3}]$ & 5 & 2 & 0 & 0 & 0 & 0 \\
\hline $\mathbf{( 0 . 3 , 0 . 4}]$ & 11 & 0 & 0 & 0 & 0 & 0 \\
\hline $\mathbf{( 0 . 4 , 0 . 5}]$ & 14 & 9 & 1 & 0 & 0 & 0 \\
\hline $\mathbf{( 0 . 5 , 0 . 6}]$ & 33 & 18 & 9 & 0 & 0 & 0 \\
\hline $\mathbf{( 0 . 6 , 0 . 7}]$ & 51 & 51 & 18 & 5 & 1 & 1 \\
\hline $\mathbf{( 0 . 7 , 0 . 8}]$ & 52 & 51 & 51 & 28 & 21 & 15 \\
\hline $\mathbf{( 0 . 8 , 0 . 9}]$ & 52 & 63 & 78 & 92 & 81 & 74 \\
\hline $\mathbf{( 0 . 9 , 1 . 0}]$ & 53 & 76 & 100 & 146 & 149 & 175 \\
\hline $\mathbf{( 1 . 0 , 1 . 1 ]}$ & 50 & 85 & 91 & 104 & 151 & 159 \\
\hline $\mathbf{( 1 . 1 , 1 . 2}]$ & 48 & 62 & 76 & 82 & 68 & 59 \\
\hline $\mathbf{( 1 . 2 , 1 . 3 ]}$ & 36 & 26 & 39 & 34 & 25 & 17 \\
\hline $\mathbf{( 1 . 3 , 1 . 4}]$ & 31 & 23 & 25 & 5 & 4 & 0 \\
\hline $\mathbf{( 1 . 4 , 1 . 5}]$ & 19 & 24 & 9 & 4 & 0 & 0 \\
\hline $\mathbf{( 1 . 5 , 1 . 6}]$ & 16 & 4 & 3 & 0 & 0 & 0 \\
\hline $\mathbf{( 1 . 6 , 1 . 7}]$ & 8 & 5 & 0 & 0 & 0 & 0 \\
\hline $\mathbf{( 1 . 7 , 1 . 8}]$ & 8 & 1 & 0 & 0 & 0 & 0 \\
\hline $\mathbf{( 1 . 8 , 1 . 9}]$ & 1 & 0 & 0 & 0 & 0 & 0 \\
\hline $\mathbf{( 1 . 9 , 2 . 0}]$ & 3 & 0 & 0 & 0 & 0 & 0 \\
\hline $\mathbf{( 2 . 0 , 2 . 1}]$ & 0 & 0 & 0 & 0 & 0 & 0 \\
\hline $\mathbf{( 2 . 1 , 2 . 2}]$ & 0 & 0 & 0 & 0 & 0 & 0 \\
\hline $\mathbf{( 2 . 2 , 2 . 3}]$ & 2 & 0 & 0 & 0 & 0 & 0 \\
\hline
\end{tabular}




\section{Table (4)}

The Behavior of The Average, Variance, The Mean Absolute Deviation and Mean square Error of The Bayesian estimates of Simulation II Where $\sigma^{2}=1.0, \theta_{1}=0.5, \theta_{2}=-0.6$

\begin{tabular}{|c|c|c|c|c|}
\hline $\mathbf{n}$ & AVR & VAR & MAD & MSE \\
\hline $\mathbf{2 0}$ & 0.965174 & 0.141606 & 0.290907 & 0.131695 \\
\hline $\mathbf{3 0}$ & 0.975636 & 0.081442 & 0.205158 & 0.066750 \\
\hline $\mathbf{5 0}$ & 0.999221 & 0.046067 & 0.157016 & 0.038065 \\
\hline $\mathbf{1 0 0}$ & 0.999372 & 0.021456 & 0.114107 & 0.020403 \\
\hline $\mathbf{1 5 0}$ & 0.998108 & 0.013935 & 0.094821 & 0.014100 \\
\hline $\mathbf{2 0 0}$ & 0.995855 & 0.010284 & 0.082762 & 0.011014 \\
\hline
\end{tabular}

Table (5)

Frequency Distributions of The Posterior Expectation For Simulation III where : $\sigma^{2}=2.0, \theta_{1}=0.5, \theta_{2}=-0.6$

\begin{tabular}{|c|c|c|c|c|c|c|}
\hline Intervals & $\mathbf{n}=\mathbf{2 0}$ & $\mathbf{3 0}$ & $\mathbf{5 0}$ & $\mathbf{1 0 0}$ & $\mathbf{1 5 0}$ & $\mathbf{2 0 0}$ \\
\hline $\mathbf{( . . . . , 0 . 5}]$ & 12 & 2 & 0 & 0 & 0 & 0 \\
\hline $\mathbf{( 0 . 1 , 0 . 2 ]}$ & 1 & 0 & 0 & 0 & 0 & 0 \\
\hline $\mathbf{( 0 . 2 , 0 . 3 ]}$ & 0 & 0 & 0 & 0 & 0 & 0 \\
\hline $\mathbf{( 0 . 3 , 0 . 4 ]}$ & 2 & 0 & 0 & 0 & 0 & 0 \\
\hline $\mathbf{( 0 . 4 , 0 . 5}]$ & 1 & 1 & 0 & 0 & 0 & 0 \\
\hline $\mathbf{( 0 . 5 , 0 . 6}]$ & 3 & 0 & 0 & 0 & 0 & 0 \\
\hline $\mathbf{( 0 . 6 , 0 . 7 ]}$ & 0 & 0 & 0 & 0 & 0 & 0 \\
\hline $\mathbf{( 0 . 7 , 0 . 8}]$ & 5 & 3 & 0 & 0 & 0 & 0 \\
\hline $\mathbf{( 0 . 8 , 0 . 9}]$ & 9 & 1 & 0 & 0 & 0 & 0 \\
\hline $\mathbf{( 0 . 9 , 1 . 0 ]}$ & 14 & 3 & 0 & 0 & 0 & 0 \\
\hline $\mathbf{( 1 . 0 , 1 . 1 ]}$ & 11 & 6 & 1 & 0 & 0 & 0 \\
\hline $\mathbf{( 1 . 1 , 1 . 2 ]}$ & 17 & 12 & 4 & 0 & 0 & 0 \\
\hline $\mathbf{( 1 . 2 , 1 . 3}]$ & 18 & 21 & 8 & 1 & 0 & 0 \\
\hline
\end{tabular}




\begin{tabular}{|c|c|c|c|c|c|c|}
\hline Intervals & $\mathbf{n}=\mathbf{2 0}$ & 30 & 50 & 100 & 150 & 200 \\
\hline$(1.3,1.4]$ & 23 & 22 & 11 & 3 & 0 & 0 \\
\hline$(1.4,1.5]$ & 26 & 16 & 20 & 10 & 6 & 3 \\
\hline$(1.5,1.6]$ & 30 & 38 & 38 & 23 & 6 & 4 \\
\hline$(1.6,1.7]$ & 27 & 36 & 37 & 42 & 28 & 23 \\
\hline$(1.7,1.8]$ & 36 & 47 & 41 & 49 & 65 & 48 \\
\hline$(1.8,1.9]$ & 21 & 39 & 54 & 57 & 76 & 75 \\
\hline$(1.9,2.0]$ & 29 & 42 & 41 & 75 & 83 & 92 \\
\hline$(2.0,2.1]$ & 21 & 33 & 41 & 72 & 69 & 93 \\
\hline$(2.1,2.2]$ & 32 & 20 & 51 & 43 & 61 & 70 \\
\hline$(2.2,2.3]$ & 16 & 21 & 35 & 46 & 35 & 35 \\
\hline$(2.3,2.4]$ & 19 & 15 & 26 & 27 & 27 & 37 \\
\hline$(2.4,2.5]$ & 18 & 28 & 27 & 20 & 30 & 14 \\
\hline$(2.5,2.6]$ & 17 & 27 & 19 & 11 & 11 & 4 \\
\hline$(2.6,2.7]$ & 12 & 10 & 9 & 10 & 0 & 1 \\
\hline$(2.7,2.8]$ & 12 & 9 & 9 & 3 & 2 & 0 \\
\hline$(2.8,2.9]$ & 10 & 10 & 9 & 4 & 0 & 1 \\
\hline$(2.9,3.0]$ & 11 & 7 & 7 & 1 & 1 & 0 \\
\hline$(3.0,3.1]$ & 8 & 7 & 4 & 2 & 0 & 0 \\
\hline$(3.1,3.2]$ & 8 & 3 & 1 & 0 & 0 & 0 \\
\hline$(3.2,3.3]$ & 11 & 7 & 3 & 0 & 0 & 0 \\
\hline$(3.3,3.4]$ & 4 & 4 & 3 & 1 & 0 & 0 \\
\hline$(3.4,3.5]$ & 5 & 3 & 0 & 0 & 0 & 0 \\
\hline$(3.5,3.6]$ & 3 & 2 & 1 & 0 & 0 & 0 \\
\hline$(3.6,3.7]$ & 0 & 1 & 0 & 0 & 0 & 0 \\
\hline$(3.7,3.8]$ & 3 & 1 & 0 & 0 & 0 & 0 \\
\hline$(3.8,3.9]$ & 3 & 2 & 0 & 0 & 0 & 0 \\
\hline$(3.9,4.0]$ & 2 & 1 & 0 & 0 & 0 & 0 \\
\hline
\end{tabular}


Table (6)

The Behavior of The Average, Variance, The Mean Absolute Deviation and Mean square Error of The Bayesian estimates of Simulation III Where $\sigma^{2}=2.0, \theta_{1}=0.5, \theta_{2}=-0.6$

\begin{tabular}{|c|c|c|c|c|}
\hline $\mathbf{n}$ & AVR & VAR & MAD & MSE \\
\hline 20 & 2.003304 & 0.621265 & 0.619673 & 0.646269 \\
\hline 30 & 2.006890 & 0.350515 & 0.460663 & 0.353876 \\
\hline 50 & 2.026310 & 0.190740 & 0.341605 & 0.186406 \\
\hline 100 & 2.013410 & 0.087327 & 0.238347 & 0.094410 \\
\hline 150 & 2.011745 & 0.056666 & 0.199388 & 0.061293 \\
\hline $\mathbf{2 0 0}$ & 2.015842 & 0.042139 & 0.168944 & 0.045172 \\
\hline
\end{tabular}

Table (7)

Frequency Distributions of The Posterior Expectation For Simulation IV where : $\sigma^{2}=0.5, \theta_{1}=0.5, \theta_{2}=0.3$

\begin{tabular}{|c|c|c|c|c|c|c|}
\hline Intervals & $\mathbf{n = 2 0}$ & $\mathbf{3 0}$ & $\mathbf{5 0}$ & $\mathbf{1 0 0}$ & $\mathbf{1 5 0}$ & $\mathbf{2 0 0}$ \\
\hline $\mathbf{( - 0 . 5 , - 0 . 4 ]}$ & 0 & 0 & 0 & 0 & 0 & 0 \\
\hline $\mathbf{( - 0 . 4 , - 0 . 3 ]}$ & 0 & 0 & 0 & 0 & 0 & 0 \\
\hline $\mathbf{( - 0 . 3 , - 0 . 2 ]}$ & 0 & 0 & 0 & 0 & 0 & 0 \\
\hline $\mathbf{( - 0 . 2 , - 0 . 1 ]}$ & 0 & 0 & 0 & 0 & 0 & 0 \\
\hline $\mathbf{( - 0 . 1 , 0 . 0 ]}$ & 1 & 0 & 0 & 0 & 0 & 0 \\
\hline $\mathbf{( 0 . 0 , 0 . 1}]$ & 2 & 0 & 0 & 0 & 0 & 0 \\
\hline $\mathbf{( 0 . 1 , 0 . 2 ]}$ & 7 & 2 & 1 & 0 & 0 & 0 \\
\hline $\mathbf{( 0 . 2 , 0 . 3 ]}$ & 41 & 21 & 7 & 0 & 0 & 0 \\
\hline $\mathbf{( 0 . 3 , 0 . 4 ]}$ & 97 & 100 & 64 & 43 & 20 & 11 \\
\hline $\mathbf{( 0 . 4 , 0 . 5}]$ & 101 & 132 & 174 & 196 & 226 & 255 \\
\hline $\mathbf{( 0 . 5 , 0 . 6}]$ & 106 & 111 & 142 & 213 & 231 & 219 \\
\hline $\mathbf{( 0 . 6 , 0 . 7 ]}$ & 65 & 76 & 81 & 47 & 23 & 15 \\
\hline $\mathbf{( 0 . 7 , 0 . 8}]$ & 36 & 35 & 28 & 1 & 0 & 0 \\
\hline $\mathbf{( 0 . 8 , 0 . 9}]$ & 22 & 17 & 3 & 0 & 0 & 0 \\
\hline $\mathbf{( 0 . 9 , 1 . 0 ]}$ & 11 & 6 & 0 & 0 & 0 & 0 \\
\hline
\end{tabular}




\begin{tabular}{|c|c|c|c|c|c|c|}
\hline Intervals & $\mathbf{n}=\mathbf{2 0}$ & $\mathbf{3 0}$ & $\mathbf{5 0}$ & $\mathbf{1 0 0}$ & $\mathbf{1 5 0}$ & $\mathbf{2 0 0}$ \\
\hline $\mathbf{( 1 . 0 , 1 . 1 ]}$ & 8 & 0 & 0 & 0 & 0 & 0 \\
\hline $\mathbf{( 1 . 1 , 1 . 2 ]}$ & 2 & 0 & 0 & 0 & 0 & 0 \\
\hline $\mathbf{( 1 . 2 , 1 . 3 ]}$ & 0 & & 0 & 0 & 0 & 0 \\
\hline $\mathbf{( 1 . 3 , 1 . 4 ]}$ & 1 & & 0 & 0 & 0 & 0 \\
\hline $\mathbf{( 1 . 4 , 1 . 5}]$ & 0 & & 0 & 0 & 0 & 0 \\
\hline $\mathbf{( 1 . 5 , 1 . 6}]$ & 0 & & 0 & 0 & 0 & 0 \\
\hline
\end{tabular}

\section{Table (8)}

The Behavior of The Average, Variance, The Mean Absolute Deviation and Mean square Error of The Bayesian estimates of Simulation IV Where $\sigma^{2}=0.5, \theta_{1}=0.5, \theta_{2}=0.3$

\begin{tabular}{|c|c|c|c|c|}
\hline $\mathbf{n}$ & AVR & VAR & MAD & MSE \\
\hline $\mathbf{2 0}$ & 0.518531 & 0.040869 & 0.150451 & 0.037986 \\
\hline $\mathbf{3 0}$ & 0.511705 & 0.022705 & 0.118065 & 0.022114 \\
\hline $\mathbf{5 0}$ & 0.511319 & 0.012148 & 0.086086 & 0.012010 \\
\hline $\mathbf{1 0 0}$ & 0.502032 & 0.005421 & 0.059908 & 0.005460 \\
\hline $\mathbf{1 5 0}$ & 0.501793 & 0.003520 & 0.046722 & 0.003428 \\
\hline $\mathbf{2 0 0}$ & 0.501406 & 0.002605 & 0.040651 & 0.002613 \\
\hline
\end{tabular}




\section{Table (9)}

Frequency Distributions of The Posterior Expectation For Simulation $\mathrm{V}$ where :

$$
\sigma^{2}=1.0, \theta_{1}=0.5, \theta_{2}=0.3
$$

\begin{tabular}{|c|c|c|c|c|c|c|}
\hline Intervals & $\mathbf{n}=\mathbf{2 0}$ & $\mathbf{3 0}$ & $\mathbf{5 0}$ & $\mathbf{1 0 0}$ & $\mathbf{1 5 0}$ & $\mathbf{2 0 0}$ \\
\hline $\mathbf{( - . . . , 0 . 1 ]}$ & 7 & 1 & 0 & 0 & 0 & 0 \\
\hline $\mathbf{( 0 . 1 , 0 . 2}]$ & 0 & 0 & 0 & 0 & 0 & 0 \\
\hline $\mathbf{( 0 . 2 , 0 . 3}]$ & 2 & 0 & 0 & 0 & 0 & 0 \\
\hline $\mathbf{( 0 . 3 , 0 . 4 ]}$ & 6 & 1 & 1 & 0 & 0 & 0 \\
\hline $\mathbf{( 0 . 4 , 0 . 5}]$ & 15 & 5 & 0 & 0 & 0 & 0 \\
\hline $\mathbf{( 0 . 5 , 0 . 6}]$ & 39 & 25 & 4 & 0 & 0 & 0 \\
\hline $\mathbf{( 0 . 6 , 0 . 7 ]}$ & 41 & 38 & 21 & 6 & 2 & 1 \\
\hline $\mathbf{( 0 . 7 , 0 . 8}]$ & 52 & 68 & 63 & 26 & 13 & 5 \\
\hline $\mathbf{( 0 . 8 , 0 . 9}]$ & 64 & 75 & 80 & 86 & 78 & 71 \\
\hline $\mathbf{( 0 . 9 , 1 . 0 ]}$ & 53 & 59 & 95 & 142 & 171 & 167 \\
\hline $\mathbf{( 1 . 0 , 1 . 1}]$ & 48 & 62 & 86 & 120 & 136 & 176 \\
\hline $\mathbf{( 1 . 1 , 1 . 2 ]}$ & 41 & 41 & 66 & 71 & 62 & 62 \\
\hline $\mathbf{( 1 . 2 , 1 . 3 ]}$ & 23 & 42 & 40 & 31 & 30 & 14 \\
\hline $\mathbf{( 1 . 3 , 1 . 4}]$ & 34 & 33 & 25 & 14 & 6 & 4 \\
\hline $\mathbf{( 1 . 4 , 1 . 5}]$ & 16 & 22 & 11 & 4 & 2 & 0 \\
\hline $\mathbf{( 1 . 5 , 1 . 6}]$ & 17 & 14 & 5 & 0 & 0 & 0 \\
\hline $\mathbf{( 1 . 6 , 1 . 7 ]}$ & 19 & 9 & 2 & 0 & 0 & 0 \\
\hline $\mathbf{( 1 . 7 , 1 . 8}]$ & 10 & 4 & 1 & 0 & 0 & 0 \\
\hline $\mathbf{( 1 . 8 , 1 . 9}]$ & 9 & 1 & 0 & 0 & 0 & 0 \\
\hline $\mathbf{( 1 . 9 , 2 . 0}]$ & 4 & 0 & 0 & 0 & 0 & 0 \\
\hline $\mathbf{( 2 . 0 , 2 . 1}]$ & 0 & 0 & 0 & 0 & 0 & 0 \\
\hline
\end{tabular}




\section{Table (10)}

The Behavior of The Average, Variance, The Mean Absolute Deviation and Mean square Error of The Bayesian estimates of

Simulation V Where $\sigma^{2}=1.0, \theta_{1}=0.5, \theta_{2}=0.3$

\begin{tabular}{|c|c|c|c|c|}
\hline $\mathbf{n}$ & AVR & VAR & MAD & MSE \\
\hline $\mathbf{2 0}$ & 1.022794 & 0.159007 & 0.303468 & 0.146966 \\
\hline $\mathbf{3 0}$ & 0.997940 & 0.086446 & 0.236927 & 0.084700 \\
\hline $\mathbf{5 0}$ & 1.000185 & 0.046405 & 0.167032 & 0.043754 \\
\hline $\mathbf{1 0 0}$ & 1.004963 & 0.021713 & 0.113722 & 0.021420 \\
\hline $\mathbf{1 5 0}$ & 1.007261 & 0.014202 & 0.096896 & 0.015147 \\
\hline $\mathbf{2 0 0}$ & 1.004057 & 0.010451 & 0.081227 & 0.010826 \\
\hline
\end{tabular}

\section{Table (11)}

Frequency Distributions of The Posterior Expectation For Simulation VI where :

$\sigma^{2}=2.0, \theta_{1}=0.5, \theta_{2}=0.3$

\begin{tabular}{|c|c|c|c|c|c|c|}
\hline Intervals & $\mathbf{n = 2 0}$ & $\mathbf{3 0}$ & $\mathbf{5 0}$ & $\mathbf{1 0 0}$ & $\mathbf{1 5 0}$ & $\mathbf{2 0 0}$ \\
\hline $\mathbf{( . . , 0 . 8}]$ & 72 & 38 & 10 & 0 & 0 & 0 \\
\hline $\mathbf{( 0 . 8 , 0 . 9}]$ & 7 & 5 & 0 & 0 & 0 & 0 \\
\hline $\mathbf{( 0 . 9 , 1 . 0 ]}$ & 10 & 3 & 1 & 0 & 0 & 0 \\
\hline $\mathbf{( 1 . 0 , 1 . 1}]$ & 8 & 3 & 2 & 0 & 0 & 0 \\
\hline $\mathbf{( 1 . 1 , 1 . 2 ]}$ & 16 & 13 & 4 & 0 & 0 & 0 \\
\hline $\mathbf{( 1 . 2 , 1 . 3 ]}$ & 16 & 14 & 11 & 4 & 0 & 0 \\
\hline $\mathbf{( 1 . 3 , 1 . 4}]$ & 28 & 20 & 15 & 3 & 1 & 0 \\
\hline $\mathbf{( 1 . 4 , 1 . 5}]$ & 25 & 25 & 14 & 10 & 1 & 0 \\
\hline $\mathbf{( 1 . 5 , 1 . 6}]$ & 29 & 21 & 29 & 11 & 10 & 7 \\
\hline $\mathbf{( 1 . 6 , 1 . 7 ]}$ & 25 & 31 & 24 & 30 & 24 & 14 \\
\hline $\mathbf{( 1 . 7 , 1 . 8}]$ & 26 & 32 & 50 & 53 & 39 & 48 \\
\hline $\mathbf{( 1 . 8 , 1 . 9}]$ & 26 & 32 & 45 & 60 & 72 & 87 \\
\hline $\mathbf{( 1 . 9 , 2 . 0}]$ & 24 & 31 & 42 & 65 & 96 & 82 \\
\hline
\end{tabular}




\begin{tabular}{|c|c|c|c|c|c|c|}
\hline Intervals & $\mathbf{n}=\mathbf{2 0}$ & $\mathbf{3 0}$ & $\mathbf{5 0}$ & $\mathbf{1 0 0}$ & $\mathbf{1 5 0}$ & $\mathbf{2 0 0}$ \\
\hline $\mathbf{( 2 . 0 , 2 . 1 ]}$ & 26 & 33 & 55 & 67 & 80 & 96 \\
\hline $\mathbf{( 2 . 1 , 2 . 2 ]}$ & 26 & 25 & 33 & 59 & 69 & 78 \\
\hline $\mathbf{( 2 . 2 , 2 . 3}]$ & 38 & 32 & 32 & 52 & 47 & 48 \\
\hline $\mathbf{( 2 . 3 , 2 . 4 ]}$ & 20 & 33 & 32 & 37 & 32 & 24 \\
\hline $\mathbf{( 2 . 4 , 2 . 5 ]}$ & 12 & 28 & 33 & 18 & 15 & 10 \\
\hline $\mathbf{( 2 . 5 , 2 . 6}]$ & 20 & 20 & 26 & 14 & 11 & 6 \\
\hline $\mathbf{( 2 . 6 , 2 . 7 ]}$ & 16 & 20 & 16 & 10 & 3 & 0 \\
\hline $\mathbf{( 2 . 7 , 2 . 8}]$ & 7 & 14 & 10 & 3 & 0 & 0 \\
\hline $\mathbf{( 2 . 8 , 2 . 9 ]}$ & 10 & 13 & 12 & 4 & 0 & 0 \\
\hline $\mathbf{( 2 . 9 , 3 . 0 ]}$ & 13 & 14 & 4 & 0 & 0 & 0 \\
\hline $\mathbf{( 3 . 0 , 3 . 1 ]}$ & 0 & 0 & 0 & 0 & 0 & 0 \\
\hline
\end{tabular}

Table (12)

The Behavior of The Average, Variance, The Mean Absolute Deviation and Mean square Error of The Bayesian estimates of Simulation VI Where $\sigma^{2}=2.0, \theta_{1}=0.5, \theta_{2}=0.3$

\begin{tabular}{|c|c|c|c|c|}
\hline $\mathbf{n}$ & AVR & VAR & MAD & MSE \\
\hline $\mathbf{2 0}$ & 2.081899 & 0.657345 & 0.601162 & 0.602487 \\
\hline $\mathbf{3 0}$ & 2.085906 & 0.377462 & 0.484457 & 0.374648 \\
\hline $\mathbf{5 0}$ & 2.052676 & 0.195835 & 0.348940 & 0.195576 \\
\hline $\mathbf{1 0 0}$ & 2.028975 & 0.088432 & 0.231458 & 0.084605 \\
\hline $\mathbf{1 5 0}$ & 2.023514 & 0.057171 & 0.178608 & 0.050832 \\
\hline $\mathbf{2 0 0}$ & 2.014076 & 0.042008 & 0.160334 & 0.039469 \\
\hline
\end{tabular}


Inspection of the numerical results of the tables supports the adequacy of the gamma approximation, developed by Broemeling and Shaarawy(1988), in estimating the variance parameter of the error term of the second order moving average processes.

\section{SUMMARY AND GONGLUSIONS}

The main objective of this paper is to examine the performance of the gamma approximation, proposed by Broemeling and Shaarawy (1988), to estimate the error variance of the second order moving average processes . In order to achieve the main objective, six simulation studies have been conducted with different variance values and the frequency distributions have been examined. In addition, the mean, variance, the mean absolute deviation and the mean square error have been calculated. The numerical results show that the gamma approximation can efficiently estimate the variance parameter with high precision for moderate and large sample size. 


\section{REFERENGES}

[1] Box, G. and Jenkins, G. (1970). Time Series Analysis, Forecasting and Control.(Holden-Day, San Francisco).

[2] Box, G. , Jenkins, G. and Reinsel, G. (2008). Time Series Analysis, Forecasting and Control. $\left(4^{\text {th }}\right.$ edition. Wiley, New York).

[3] Broemeling, L. and Shaarawy, S. (1986). A Bayesian Analysis of Time Series, Bayesian Inferences Decision Techniques with Applications : Essay in Honor of Bruno de Finetti, (edited by Goeland Zellener).

[4] Broemeling, L. and Shaarawy, S. (1988). Time Series Analysis : A Bayesian Analysis in the Time Domain. Bayesian Analysis of Time Series and Dynamic Model, (edited by Spall, J).

[5] Chatfield, C. (1980). The Analysis of Time Series : Theory and Practice . (Chapman and Hall Ltd., London).

[6] Harvey, A. (1993). Time Series Models, (2 ${ }^{\text {nd }}$ edition. The MIT Press).

[7] Kutbi, M. (2010). "The Efficiency of the Gamma Approximation of Bayesian Estimation of the Error Precision for the First Order Moving Average Process". The Scientific Journal of Commerce Faculty, Azhar University, Vol.6.

[8] Lahif, M. (1980). "Time Series Forecasting with noninformative Prior Distribution". Technical Report No.111, Department of Statistics, University of Chicago.

[9] Liu, L. (2009), Time Series Analysis and Forecasting. (2 ${ }^{\text {nd }}$ edition. Scientific Computing Association Corp,USA).

[10] Macleod, A. (1977). "Improved Box-Jenkins Estimators". Biometrika, Vol. 64, pp. 531-534.

[11] Monahan, J. (1983). "Fully Bayesian Analysis of ARIMA Time Series Models", Journal of Econometrics, Vol. 21, pp. 307-331. 
[12] Newbold, P.(1973). "Bayesian Estimation of Box and Jenkins Transfer Function Model for Noise Models". Journal of the Royal Statistical Society, Series B, vol. 35. No. 2 , pp. 323-336.

[13] Phadke, M.S. and Kedem, G. (1978). "Computation of the exact Likelihood Function of Multivariate Moving Average Models". Biometrika, vol. 65, pp. 511-519.

[14] Priestely, M. (1981). Spectral Analysis of Time Series , (Academic Press, London).

[15] Shaarawy, S. and Broemeling, L. (1984). "Bayesian Inference and Forecasts with Moving Average Processes" . Comm. In Statis. Vol.13, No. 15, pp. 1871-1888.

[16] Shaarawy, S. and El-Shawadfy, G. (1994). "On the Adequacy of using T Approximation in Bayesian Inferences of ARMA Models". The Egyption Statistical Journal Institute of Statistical Studies and Research, Cairo university.

[17] Wei, W.W.S. (2005). Time Series Analysis : Univariate and Multivariate Methods . (Addison Wesley, Reading, MA).

[18] Zellner, A. (1971). An Introduction to Bayesian Inference in Econometrics. (John Wiley and Sons. Inc, New York).

[19] Zellner, A. and Reynolds, R. (1978). "Bayesian Analysis of ARMA Models". Presented at the Sixteenth Seminar on Bayesian Inference in Econometrics, Vol. 23, pp. 1-5, June 2-3. 